\title{
Effects of attentional focus on postural sway in children and adults
}

\author{
Isabelle Olivier $\cdot$ Estelle Palluel $\cdot$ Vincent Nougier
}

Received: 1 August 2007/Accepted: 4 January 2008/Published online: 24 January 2008

(c) Springer-Verlag 2008

\begin{abstract}
The present study examined, in children aged 4-11 and in adults, the postural control modifications when attention was oriented voluntary on postural sway. Since (1) there are less attentional resources in children than in adults, (2) the selective attention processing improves with age, i.e., children use a different strategy to focus their attention than adults, and (3) adults' postural stability decreases when attention is focused on postural sway, we hypothesized that postural stability was less affected in children than in adults when attention was focused on postural sway. Fourty four children aged 4- to 11-year-old and 11 adults participated in the experiments. The postural control task was executed in a Romberg position. Two experimental conditions were presented to the subjects, (1) to look at a video on a TV screen without instruction about the posture, and (2) to fixate a cross placed at the center of the TV screen with the instruction to remain as stable as possible. Postural performance was measured by means of a force platform. Results from this study (1) confirmed a non-monotonic improvement of postural stability during the ontogenetic period without reaching the adults' level at the age of 11 , (2) suggested that children, aged 4-11, are able to focus their attention on the control of posture, and (3) showed that the automatic control of posture increases postural stability since the age of 4 .
\end{abstract}

Keywords Development - Posture - Attention . Stability

I. Olivier $(\varangle) \cdot$ E. Palluel · V. Nougier

Faculté de Médecine, Bâtiment Jean Roget,

Laboratoire TIMC-IMAG, UMR CNRS 5525,

Equipe "Santé, Plasticité, Motricité",

Joseph Fourier University,

38706 La Tronche Cedex, France

e-mail: Isabelle.Olivier@ujf-grenoble.fr

\section{Introduction}

For a long time, postural control was only considered as an automatic response (Belenkii et al. 1967). Nevertheless, some recent investigations provided evidence that the regulation of posture, from the simplest task (i.e., orthostatic) to the most complex one (i.e., unipodal balance), also involves attentional processes (Woollacott and Shumway-Cook 2002; Vuillerme and Nougier 2004). The mobilization of these attentional resources depends on (1) the age (Teasdale and Simoneau 2001; Woollacott and Shumway-Cook 2002), (2) the available sensory information (Shumway-Cook and Woollacott 2000; Teasdale and Simoneau 2001), (3) the postural task complexity (Lajoie et al. 1996), (4) the expertise (Vuillerme and Nougier 2004), and (5) the voluntary attentional focus on body sway (Vuillerme and Nafati 2007). Indeed, these last authors observed a degradation of postural stability when it was asked to adult subjects to focus their attention on their posture. One explanation of this degradation was that focusing attention on the movement implies an increase of the voluntary control which then comes to interfere with the automatic processes usually used to realize it (Wulf and Prinz 2001). From a neurological point of view, the degradation observed when voluntary attention focused on the movement (Zachry et al. 2005) or on body sway (Vuillerme and Nafati 2007) was associated with an increase of the neuromuscular activity. These last authors interpreted this modulation of neuromuscular activity by the fact that voluntary motor control increases the recruitment of additional motor units, inducing an increase of the muscular force which in turn degrades the control of upright stance.

In children, it has been shown that the attentional capacity increases as age increases (Wickens 1974). Moreover, studies investigating the development of 
selective attention, i.e., the ability to attend voluntarily to some attributes of the stimulus array while ignoring other attributes (Enns and Girgus 1984), have suggested that the efficiency of this attentional mechanism improves during childhood. Regarding postural capacity, most of the authors observed an improvement of postural stability during childhood (Kirshenbaum et al. 2001; Sundermier et al. 2001). For example, Rival et al. (2005) showed that the maximal amplitude of the center of foot pressure (CoP) decreases in a non-monotonic way, and that the mean speed of the CoP decreases linearly between 6 and 10 years of age. The existence of a critical period around the age of 7 , characterized by a change of postural strategy looking more and more similar to that adopted by the adults, is now well established (Assaiante and Amblard 1995). Nevertheless, although the development of balance control has generated significant interest, investigations of the corresponding increase in attentional resources remain scarce. To our knowledge, only two recent studies investigated the interacting effects of a cognitive and postural task in children (Blanchard et al. 2005; Olivier et al. 2007). The authors concluded that concurrent cognitive tasks while standing affected postural sway in children aged 7 and 9.5 years. However, to our knowledge, no study investigated in children to which extent posture is controlled through automatic processes as in adults. Moreover, no authors studied in children the capacity to modulate the neuromuscular activity of the lower limb muscles required for controlling upright stance as showed in adults ( $\mathrm{Vu}-$ illerme and Nafati 2007).

The purpose of the present study was to assess whether the decrease of postural stability observed in adults when attention is focused on postural sway was also observed in children aged 4-11. We hypothesized that postural stability was less affected by the focusing of attention on postural sway in children than in adults.

\section{Materials and methods}

\section{Subjects}

Fifty five subjects participated in the experiment. Four groups of 11 children: 4-5 years old (seven males and four females, $M=4.8$ years, $\mathrm{SD}=5$ months); $6-7$ years old (six males and five females, $M=6.4$ years, $\mathrm{SD}=6$ months); $8-9$ years old (three males and eight females, $M=8.9$ years, $\mathrm{SD}=6$ months) and $10-11$ years old (10 males and 1 female, $M=10.7$ years, $\mathrm{SD}=10$ months), and a group of 11 adults: 26 years old (four males and seven females, $M=25$ years, $\mathrm{SD}=14$ months). All participants were naive as to the purpose of the experiment, had a normal scholastic level, and did not show any known neurological or motor disorders. This study was approved by the local ethics committee and in conformity with the Helsinki Convention informed consent was obtained from all subjects.

\section{Experimental set-up}

Subjects stood barefoot in a Romberg position with their arms hanging loosely by their sides, and their feet were placed slightly apart $(14 \mathrm{~cm})$ on marks drawn on the force platform (AMTI ${ }^{\circledR}$, model OR6-5-1). A television (TV) was placed $150 \mathrm{~cm}$ in front of the subjects with the center of the screen aligned on the middle of the body, at the eye level. Two experimental conditions were presented to the subjects, (1) to look at a video (i.e., a cartoon) on the TV screen without instruction about the posture and (2) to fixate a stationary cross placed at the center of the TV screen. In this experimental condition, subjects were asked to remain as stable as possible for the complete duration of the trial. Whatever the block, a trial had $30 \mathrm{~s}$ of duration. The two experimental blocks, composed of five trials each, were randomized among subjects. The time interval between two blocks was $5 \mathrm{~min}$, approximately. None of the subjects complained about fatigue. Before each trial, subjects were given a verbal preparatory signal ("ready") by the experimenter, and, approximately $3 \mathrm{~s}$ later, the experimenter said "go". At the end of trial a third verbal signal was given to the subjects by the experimenter: "it's finished".

\section{Data processing}

Signals from the force platform were recorded at a frequency of $100 \mathrm{~Hz}$ (12 bit A/D resolution). These force plate data were filtered with a $50 \mathrm{~Hz}$ low-pass, second order Butterworth filter. The displacements of the center of foot pressure in the medio-lateral $\left(\mathrm{CoP}_{x}\right)$ and antero-posterior $\left(\mathrm{CoP}_{y}\right)$ planes were calculated using the following approximation: $\Delta \mathrm{CoP}_{x}=\Delta M_{y} / F z$ and $\Delta \mathrm{Co}_{y}=\Delta M_{x} / F z$, respectively, in which $\Delta M_{y}$ and $\Delta M_{x}$ was a change of the torque with respect to its baseline value defined as the average value within the time interval from 0 to $30 \mathrm{~s}$ (Slijper et al. 2002; Slijper and Latash 2004).

Two measures of sway were used to describe subjects' postural behavior. The range, i.e., the magnitude of the CoP (Odendrick et al. 1984), indicated the maximal excursion of the CoP in any direction. It is a global measure allowing to estimate overall postural stability. The vector sum was used to calculate the distance between two different points (formula $\sqrt{\left(x_{2}-x_{1}\right)^{2}+\left(y_{2}-y_{1}\right)^{2}}$ ), independently from the direction: The range corresponded to the greatest distance between two points of the COP 
displacement. The speed of the CoP, i.e., the frequency of the CoP (Hayes et al. 1985), indicated the mean speed of the CoP displacements over the sampled period, that is, the sum of the displacement scalars over the sampling period divided by the sampling time. This measure has been suggested to represent the amount of activity required to maintain stability (Maki et al. 1990), providing a more functional approach of posture.

\section{Statistical analysis}

To explore the effect of video on postural performance during the ontegenetic period, a 5 Ages $(4-5,6-7,8-9,10-$ 11 and 26 years) $\times 2$ Attentional conditions (with (i.e., to fixate a cross) and without (i.e., to look a video) attention focused on the posture) analysis of variance (ANOVA) with repeated measures on the last factor was applied to the range and speed of the CoP. The level of significance was set at $P<0.05$.

\section{Results}

For the range, the ANOVA showed a main effect of Age, $F_{(4,50)}=9, \quad P<0.05$. Post-hoc Newman-Keuls tests revealed that the range was larger at 4-5 years than at the others ages $(P<0.05)$ and smaller in adults than at the others ages $(P<0.05)$. As illustrated in Fig. 1, the range decreased between $4-5$ years $(36.8 \pm 5) \mathrm{mm}$ and $6-$ 11 years $(20.2 \pm 7) \mathrm{mm}$. Moreover, the adult level $(16 \pm 5) \mathrm{mm}$ was not already attained at $10-11$ years of age $(27.7 \pm 12) \mathrm{mm}$. No effect of the Attentional condition and no significant Age $\times$ Attentional condition interaction were found.

For the speed, the ANOVA showed a main effect of Age, $F_{(4,50)}=11.88, P<0.05$. Again, post-hoc Newman-

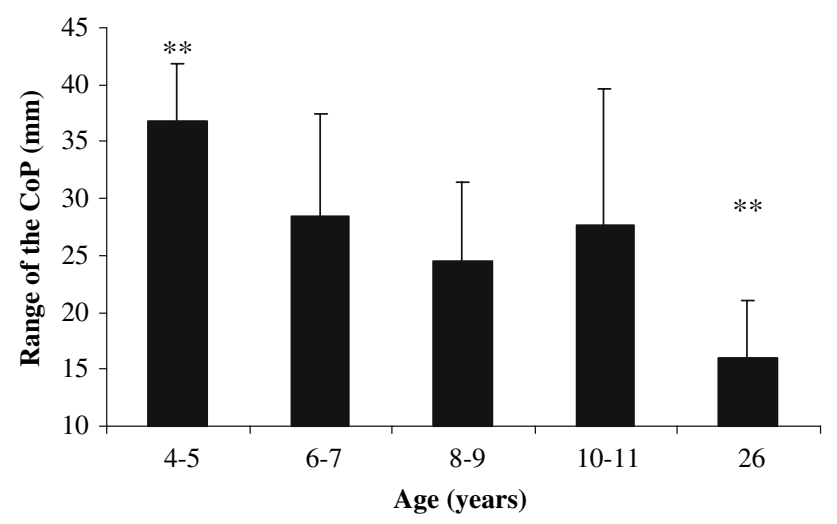

Fig. 1 Mean and standard deviation for the range $(\mathrm{mm})$ of the $\mathrm{CoP}$ as a function of age $(4-5,6-7,8-9,10-11$ and 26 years)
Keuls analysis revealed that the speed was higher at 4 5 years than at the others ages $(\mathrm{P}<0.05)$ and slower in adults than at the others ages $(\mathrm{P}<0.05)$. As illustrated in Fig. 2a, the speed: (1) decreased between 4 and 5 years $(17.4 \pm 1) \mathrm{mm} / \mathrm{s}$ and $6-7$ years $(13.3 \pm 4) \mathrm{mm} / \mathrm{s}$, (2) reached a plateau around the ages of 6-11 years, and (3) decreased between 10 and 11 years $(13,9 \pm 5) \mathrm{mm} / \mathrm{s}$ and the adult age $(6.4 \pm 1) \mathrm{mm} / \mathrm{s}$. Finally, the ANOVA also showed a main effect of Attentional condition, $\mathrm{F}_{(1,50)}=4.53, \mathrm{P}<0.05$. The CoP speed was higher in the condition with (i.e., to fixate a cross) than without (i.e., to look a video) attention focused on posture (with: $12.7 \pm 5.1 \mathrm{~mm} / \mathrm{s}$ and without: $12.2 \pm 5.2 \mathrm{~mm} / \mathrm{s}$ ) (Fig. 2 b). No significant Age $\times$ Attentional condition interaction was found.

\section{Discussion}

The aim of the present study was to investigate, in children aged $4-11$, the decrease of postural stability observed in adults in which attention was focused on postural sway. Our assumption was that children were less affected by the focusing of attention on postural sway than adults.
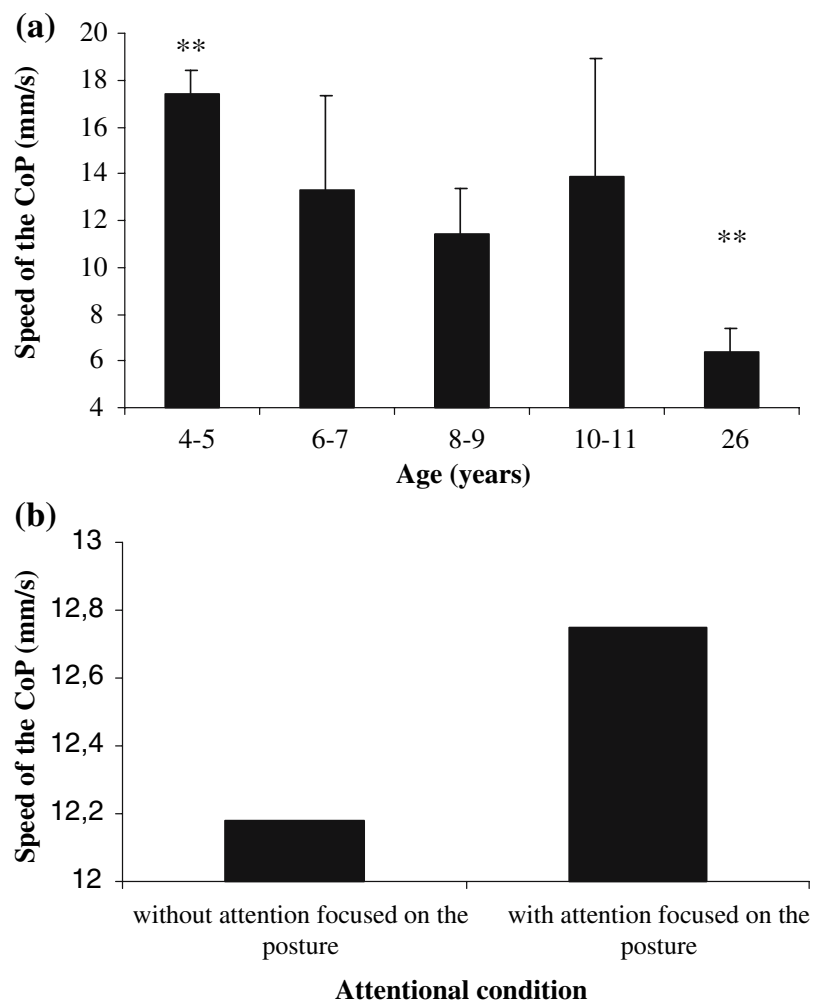

Fig. 2 Mean and standard deviation for the speed $\left(\mathrm{mm} \mathrm{s}^{-1}\right)$ of the CoP as a function of a age (4-5, 6-7, 8-9, 10-11 and 26 years), and $\mathbf{b}$ attentional condition (with and without attention focused on the posture) 
Age-related difference

In accordance with the literature (Woollacott et al. 1987; Sundermier et al. 2001; Rival et al. 2005), the present results showed a non-monotonic improvement of postural stability during childhood. More specifically, maximal amplitude and mean velocity of $\mathrm{CoP}$ displacements decreased between 4-5 and 6-7 years, reached a plateau around the ages of 6-11 years, and decreased again between 10-11 years and adult age. These results confirmed that (1) the improvement of postural control during childhood is characterized by a decreasing magnitude (i.e., Hatzitaki et al. 2002), and frequency (i.e., Figura et al. 1991) of postural sway, (2) the period of 8-11 years can be considered as a critical period (Rival et al. 2005) resulting from an integration of the reactive and predictive modes of postural control (Riach and Starkes 1994), from a better integration of sensory information (Baumberger et al. 2004), and from the apparition of adult-like balance control strategies characterized by a head-trunk coordination (Assaiante and Amblard 1995), and (3) the adults' level is still not reached at the age of 11 .

Effect of the attentional focus on postural sway according to age

The speed of CoP displacements significantly increased when subjects, whatever their age, had to fixate a cross with the instruction to remain as stable as possible. This confirmed in children as in adults that postural stability decreases when attention is focused on postural sway, which may be explained by an increase of the neuromuscular activity (Vuillerme and Nafati 2007; Wulf and Prinz 2001; Zachry et al. 2005). At least since the age of 4, focusing attention on the control of upright stance would increase the number of motor units recruited in the lower limb muscles that are involved. This explanation also allowed understanding why focusing attention on postural sway only affected speed and not magnitude of the CoP oscillations. With attention, an increased number of motor units involved in the regulation of posture probably introduced some "noise" in the postural system. This noise can be better observable with $\mathrm{CoP}$ speed than CoP range, CoP speed being a more sensitive measure to estimate the amount of activity required for maintaining postural stability. Moreover, this result suggested that children aged 411 are already able to focus their attention on the control of posture. This is in accordance with studies investigating the development of selective attention in which a benefit was systematically observed when children between 5 and 9 years of age voluntarily focused their attention onto some attributes of the stimulus array (Akhtar and Enns 1989;
Enns and Brodeur 1989). The present results showed, as in adults, the existence of a cost when children oriented their attention on some attributes of the motor response, at least since the age of 4 . Finally, this study showed that the automatic control of posture increases postural stability as soon as the age of 4 years. Future investigations are necessary to know the existence of this phenomenon before the age of 4 .

In conclusion, even though postural control processing is not yet mature at the age of 11 years, a decrease of postural stability is observed in children as in adults when too many attentional resources are mobilized to control posture. Indeed, these attentional resources seem to interfere with the automatic processes usually involved in the regulation of posture in simple tasks, such as an orthostatic condition.

Acknowledgements Authors are grateful to the children and to their parents for their precious collaboration.

\section{References}

Akhtar N, Enns JT (1989) Relations between covert orienting and filtering in the development of visual attention. J Exp Child Psychol 48:315-334

Assaiante C, Amblard B (1995) An ontogenetic model for the sensorimotor organisation of balance control in humans. Hum Mov Sci 14:13-43

Baumberger B, Isableu B, Fluckiger M (2004) The visual control of stability in children and adults: postural readjustments in a ground optical flow. Exp Brain Res 159:33-46

Belenkii VE, Gurfinkel VS, Paltsev EI (1967) On elements of control of voluntary movements. Biofizica 12:135-141

Blanchard Y, Carey S, Coffey J, Cohen A, Michlik S, Pellecchia GL (2005) The influence of concurrent cognitive tasks on postural sway in children. Pediatr Phys Ther 17:189-193

Enns JT, Brodeur DA (1989) A developmental study of covert orienting to peripheral visual cues. J Exp Child Psychol 48:171189

Enns JT, Girgus JS (1984) Developmental changes in selective and integrative visual attention. J Exp Child Psychol 40:319-337

Figura F, Cama G, Capranica L, Guidetti L, Pulejo C (1991) Assessment of static balance in children. J Sports Med Phys Fitness 31:235-242

Hatzitaki V, Zisi V, Kollias I, Kioumourtzoglou E (2002) Perceptualmotor contributions to static and dynamic balance control in children. J Mot Behav 34:161-170

Hayes KC, Spencer JD, Riach CL, Lucy SD, Kirshen AJ (1985) Age related changes in postural sway. In: Winter D, Hayes K, Patla A (eds) Biomecanics IX-A. Human Kinetics Publishers, Champlain, pp 383-387

Kirshenbaum N, Riach CL, Starkes JL (2001) Non-linear development of postural control and strategy use in young children: a longitudinal study. Exp Brain Res 140:420-431

Lajoie Y, Teasdale N, Bard C, Fleury M (1996) Upright standing and gait: are there changes in attentional requirements related to normal aging? Exp Aging Res 22:185-198

Maki B, Holliday P, Fernie G (1990) Aging and postural control: a comparaison of spontaneous- and induced-way balance tests. J Am Geriatr Soc 38:1-9 
Odendrick P, Sandstedt P (1984) Development of postural sway in the normal child. Hum Neurobiol 3:241-244

Olivier I, Cuisinier C, Vaugoyeau M, Nougier V, Assaiante C (2007) Dual-task study of cognitive and postural interference in 7-yearolds and adults. Neuroreport 18:817-821

Riach CL, Starkes JL (1994) Velocity of center of pressure excursions as an indicator of postural control systems in children. Gait Posture 2:167-172

Rival C, Ceyte H, Olivier I (2005) Developmental changes of static standing balance in children. Neurosci Lett 376:133-136

Shumway-Cook A, Woollacott MH (2000) Attentional demands and postural control: the effect of sensory context. J Gerontol Series A Biol Sci Med Sci 55:M10-M16

Slijper H, Latash ML, Mordkoff JT (2002) Anticipatory postural adjustments under simple and choice reaction time conditions. Brain Res 924:184-197

Slijper H, Latash ML (2004) The effects of muscle vibration on anticipatory postural adjustments. Brain Res 1015(1-2):57-72

Sundermier L, Woollacott M, Roncesvalles N, Jensen J (2001) The development of balance control in children: comparisons of EMG and kinetic variables and chronological and developmental groupings. Exp Brain Res 136:340-350
Teasdale N, Simoneau M (2001) Attentional demands for postural control: the effects of aging and sensory reintegration. Gait Posture 14:203-210

Vuillerme N, Nafati G (2007) How attentional focus on body sway affects postural control during quiet standing? Psychol Res 71(2):192-200

Vuillerme N, Nougier V (2004) Attentional demand for regulating postural sway: the effect of expertise in gymnastics. Brain Res Bull 63:161-165

Wickens CD (1974) Temporal limits of human information processing: a developmental study. Psychol Bull 81:739-755

Woollacott M, Debû B, Mowatt M (1987) Neuromuscular control of posture in the infant and child: is vision dominant? J Mot Behav 19:167-186

Woollacott MH, Shumway-Cook A (2002) Attention and the control of posture and gait: a review of an emerging area of research. Gait Posture 16:1-14

Wulf G, Prinz W (2001) Directing attention to movement effects enhances learning: a review. Psychon Bull Rev 8:648-660

Zachry T, Wulf G, Mercer J, Bezodis N (2005) Increased movement accuracy and reduced EMG activity as the result of adopting an external focus of attention. Brain Res Bull 67(4):304-309 